Pacific

Journal of

Mathematics

\title{
COMPLEXES OF TREES AND NESTED SET COMPLEXES
}

Eva Maria Feichtner

Volume $227 \quad$ No. 2

October 2006 


\title{
COMPLEXES OF TREES AND NESTED SET COMPLEXES
}

\author{
EVA MARIA FEICHTNER
}

\begin{abstract}
We exhibit an identity of abstract simplicial complexes between the wellstudied complex of trees $T_{n}$ and the reduced minimal nested set complex of the partition lattice. We conclude that the order complex of the partition lattice can be obtained from the complex of trees by a sequence of stellar subdivisions. We provide an explicit cohomology basis for the complex of trees that emerges naturally from this context.

Motivated by these results, we review the generalization of complexes of trees to complexes of $\boldsymbol{k}$-trees by Hanlon, and we propose yet another generalization, more natural in the context of nested set complexes.
\end{abstract}

\section{Introduction}

In this article we explore the connection between complexes of trees and nested set complexes of specific lattices.

Nested set complexes appear as the combinatorial core in De Concini and Procesi's [1995] wonderful compactifications of arrangement complements. They record the incidence structure of natural stratifications and are crucial for descriptions of topological invariants in combinatorial terms. Disregarding their geometric origin, nested set complexes can be defined for any finite meet-semilattice [Feichtner and Kozlov 2004]. Interesting connections between seemingly distant fields have been established when relating the purely order-theoretic concept of nested sets to various contexts in geometry. See [Feichtner and Yuzvinsky 2004] for a construction linking nested set complexes to toric geometry, and [Feichtner and Sturmfels 2005] for an appearance of nested set complexes in tropical geometry.

This paper presents yet another setting where nested set complexes appear in a meaningful way and, this time, contribute to the toolbox of topological combinatorics and combinatorial representation theory.

Complexes of trees $T_{n}$ are abstract simplicial complexes with simplices corresponding to combinatorial types of rooted trees on $n$ labeled leaves. They made their first appearance in [Boardman 1971], in connection with $E_{\infty}$-structures in homotopy theory. Later, they were studied in [Vogtmann 1990] from the point of

MSC2000: primary 05E25; secondary 57Q05.

Keywords: complexes of phylogenetic trees, nested set complexes, cohomology bases. 
view of geometric group theory, and in [Robinson and Whitehouse 1996] from the point of view of representation theory. In fact, $T_{n}$ carries a natural action of the symmetric group $\Sigma_{n}$ that allows for a lifting to a $\Sigma_{n+1}$-action. For studying induced representations in homology, Robinson and Whitehouse determined the homotopy type of $T_{n}$ to be a wedge of $(n-1)$ ! spheres of dimension $n-3$. Later on, complexes of trees were shown to be shellable by Trappmann and Ziegler [1998] and independently by Wachs (1998; see [Wachs 2003]). Recent interest in the complexes is motivated by the study of spaces of phylogenetic trees from combinatorial, geometric and statistics point of view [Billera et al. 2001]. Complexes of trees appear as links of the origin in natural polyhedral decompositions of the spaces of phylogenetic trees.

Ardila and Klivans [2006] recently proved that the complex of trees $T_{n}$ can be subdivided by the order complex of the partition lattice $\Delta\left(\Pi_{n}\right)$. Our result shows that $\Delta\left(\Pi_{n}\right)$, in fact, can be obtained by a sequence of stellar subdivisions from the complex of trees. This and other corollaries rely on the specific properties of nested set complexes that we introduce into the picture.

Our paper is organized as follows: After recalling the definitions of complexes of trees and of nested set complexes in Section 2, we establish an isomorphism between the complex of trees $T_{n}$ and the reduced minimal nested set complex of the partition lattice $\Pi_{n}$ in Section 3. Among several corollaries, we observe that the isomorphism provides a $\Sigma_{n}$-invariant approach for studying tree complexes; their $\Sigma_{n}$-representation theory can be retrieved literally for free.

In Section 4 we complement the by now classical combinatorial correspondence between no broken circuit bases and decreasing EL-labeled chains for geometric lattices by incorporating proper maximal nested sets as defined in [De Concini and Procesi 2005]. We formulate a cohomology basis for the complex of trees that emerges naturally from this combinatorial setting.

Mostly due to their rich representation theory, complexes of trees have been generalized to complexes of homeomorphically irreducible $k$-trees in [Hanlon 1996]. We discuss this and another, in the nested set context more natural, generalization in Section 5.

\section{Main characters}

2.1. The complex of trees. Let us fix some terminology: A tree is a cycle-free graph; vertices of degree 1 are called leaves of the tree. A rooted tree is a tree with one vertex of degree larger 1 marked as the root of the tree. Vertices other than the leaves and the root are called internal vertices. We assume that internal vertices have degree at least 3 . The root of the tree is thus the only vertex that can have degree 2 . Another way of saying this is that we assume all nonleaves to 
have outdegree at least 2, where the outdegree of a vertex is the number of adjacent edges that do not lie on the unique path between the vertex and the root.

We call a rooted tree binary if the vertex degrees are minimal, i.e., the root has degree 2 and the internal vertices have degree 3 ; in other words, if the outdegree of all nonleaves is 2 . Observe that a rooted binary tree on $n$ leaves has exactly $n-2$ internal edges, i.e., edges that are not adjacent to a leave.

The combinatorial type of a rooted tree with labeled leaves refers to its equivalence class under label- and root-preserving homeomorphisms of trees as 1-dimensional cell complexes.

Rooted trees on $n$ leaves labeled with integers $1, \ldots, n$ are in one-to-one correspondence with trees on $n+1$ leaves labeled with integers $0, \ldots, n$ where all internal vertices have degree $\geq 3$. The correspondence is obtained by adding an edge and a leaf labeled 0 to the root of the tree. Though trees on $n+1$ labeled leaves seem to be the more natural, more symmetric objects, rooted trees on $n$ leaves come in more handy for the description of $T_{n}$.

Definition 2.1. The complex of trees $T_{n}, n \geq 3$, is the abstract simplicial complex with maximal simplices given by the combinatorial types of binary rooted trees with $n$ leaves labeled $1, \ldots, n$, and lower-dimensional simplices obtained by contracting at most $n-3$ internal edges.

The complex of trees $T_{n}$ is a pure $(n-3)$-dimensional simplicial complex. As we pointed out in the introduction, it is homotopy equivalent to a wedge of $(n-1)$ ! spheres of dimension $n-3$; see [Robinson and Whitehouse 1996, Theorem 1.5].

The complex $T_{3}$ consists of 3 points. For $n=4$, there are 4 types of trees, we depict labeled representatives in Figure 1. Observe that the first two correspond to 1-dimensional (maximal) simplices, whereas the other two correspond to vertices. The last two labeled trees, in fact, are the vertices of the edge corresponding to the first maximal tree. The third tree is a "vertex" of the second, and of the first.

2.2. Nested set complexes. We recall here the definition of building sets, nested sets, and nested set complexes for finite lattices as proposed in [Feichtner and Kozlov 2004].
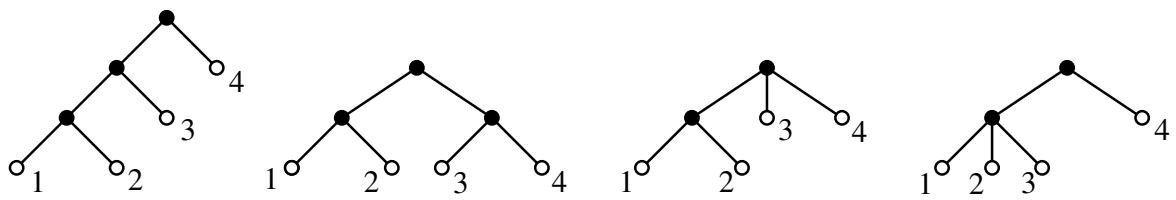

Figure 1. Simplices in $T_{4}$. 
We use the standard notation for intervals in a finite lattice $\mathscr{L}$,

$$
[X, Y]:=\{Z \in \mathscr{L} \mid X \leq Z \leq Y\}
$$

for $X, Y \in \mathscr{L}$, moreover, $\mathscr{Y}_{\leq X}:=\{Y \in \mathscr{Y} \mid Y \leq X\}$, and accordingly $\mathscr{Y}_{<X}, \mathscr{Y}_{\geq X}$, and $\mathscr{S}_{>X}$, for $\mathscr{Y} \subseteq \mathscr{L}$ and $X \in \mathscr{L}$. With $\max \mathscr{Y}$ we denote the set of maximal elements in $\mathscr{S}$ with respect to the order coming from $\mathscr{L}$.

Definition 2.2. Let $\mathscr{L}$ be a finite lattice. A subset $\mathscr{G}_{\text {in }} \mathscr{L}_{>0}$ is called a building set if for any $X \in \mathscr{L}_{>0}$ and $\max \mathscr{G}_{\leq X}=\left\{G_{1}, \ldots, G_{k}\right\}$ there is an isomorphism of partially ordered sets

$$
\varphi_{X}: \prod_{j=1}^{k}\left[\hat{0}, G_{j}\right] \stackrel{\cong}{\longrightarrow}[\hat{0}, X]
$$

with $\varphi_{X}\left(\hat{0}, \ldots, G_{j}, \ldots, \hat{0}\right)=G_{j}$ for $j=1, \ldots, k$. We call $F_{\mathscr{G}}(X):=\max \varphi_{\leq X}$ the set of factors of $X$ in $\mathscr{G}$.

The full lattice $\mathscr{L}_{>0}$ is the simplest example of a building set for $\mathscr{L}$. We will sometimes abuse notation and just write $\mathscr{L}$ in this case. Besides this maximal building set, there is always a minimal building set $\mathscr{I}$ consisting of all elements $X$ in $\mathscr{L}_{>0}$ which do not allow for a product decomposition of the lower interval $[\hat{0}, X]$, the so-called irreducible elements in $\mathscr{L}$.

Definition 2.3. Let $\mathscr{L}$ be a finite lattice and $\mathscr{G}$ a building set containing the maximal element $\hat{1}$ of $\mathscr{L}$. A subset $\mathscr{S}$ in $\mathscr{G}$ is called nested (or $\mathscr{G}$-nested if specification is needed) if, for any set of incomparable elements $X_{1}, \ldots, X_{t}$ in $\mathscr{S}$ of cardinality at least two, the join $X_{1} \vee \cdots \vee X_{t}$ does not belong to $\mathscr{G}$. The $\mathscr{G}$-nested sets form an abstract simplicial complex, $\widetilde{\mathcal{N}}(\mathscr{L}, \mathscr{G})$, the nested set complex of $\mathscr{L}$ with respect to $\mathscr{G}$. Topologically, the nested set complex is a cone with apex $\hat{1}$; its base $\mathcal{N}(\mathscr{L}, \mathscr{G})$ is called the reduced nested set complex of $\mathscr{L}$ with respect to $\mathscr{G}$.

We will mostly be concerned with reduced nested set complexes due to their more interesting topology. If the underlying lattice is clear from the context, we will write $\mathcal{N}(\mathscr{G})$ for $\mathcal{N}(\mathscr{L}, \mathscr{G})$.

Nested set complexes can be defined analogously for building sets not containing $\hat{1}$, and, even more generally, for meet semilattices. For a definition in the full generality, see [Feichtner and Kozlov 2004, Section 2].

For the maximal building set of a lattice $\mathscr{L}$, subsets are nested if and only if they are linearly ordered in $\mathscr{L}$. Hence, the reduced nested set complex $\mathcal{N}(\mathscr{L}, \mathscr{L})$ coincides with the order complex of $\mathscr{L}$, more precisely, with the order complex of the proper part, $\mathscr{L} \backslash\{\hat{0}, \hat{1}\}$, of $\mathscr{L}$, which we denote by $\Delta(\mathscr{L})$ using customary notation. 
If $\mathscr{L}$ is an atomic lattice, the nested set complexes can be realized as simplicial fans (see [Feichtner and Yuzvinsky 2004]), and for building sets $\mathscr{G} \subseteq \mathscr{H}$ in $\mathscr{L}$, the nested set complex $\widetilde{\mathcal{N}}(\mathscr{L}, \mathscr{H})$ can be obtained from $\widetilde{\mathcal{N}}(\mathscr{L}, \mathscr{G})$ by a sequence of stellar subdivisions [Feichtner and Müller 2005, Theorem 4.2]. In particular, any reduced nested set complex $\mathcal{N}(\mathscr{L}, \mathscr{G})$ is obtained by a sequence of stellar subdivisions from the minimal reduced nested set complex $\mathcal{N}(\mathscr{L}, \mathscr{I})$, and can be further subdivided by stellar subdivisions so as to obtain the maximal nested set complex $\Delta(\mathscr{L})$.

Example 2.4. Let $\Pi_{n}$ denote the lattice of set partitions of $[n]:=\{1, \ldots, n\}$ partially ordered by reversed refinement. As explained above, the reduced maximal nested set complex $\mathcal{N}\left(\Pi_{n}, \Pi_{n}\right)$ is the order complex $\Delta\left(\Pi_{n}\right)$. Irreducible elements in $\Pi_{n}$ are the partitions with exactly one nonsingleton block. They can be identified with subsets of $[n]$ of cardinality at least 2 . Nested sets for the minimal building set $\mathscr{I}$ are collections of such subsets of $[n]$ such that any two either contain one another or are disjoint. For $n=3$, the reduced minimal nested set complex consists of 3 isolated points; for $n=4$, it equals the Petersen graph.

\section{Subdividing the complex of trees}

We now state the core fact of our note.

Theorem 3.1. The complex of trees $T_{n}$ and the reduced minimal nested set complex of the partition lattice $\mathcal{N}\left(\Pi_{n}, \mathscr{I}\right)$ coincide as abstract simplicial complexes.

Proof. We exhibit a bijection between simplices in $T_{n}$ and nested sets in the reduced minimal nested set complex $\mathcal{N}\left(\Pi_{n}, \mathscr{I}\right)$ of the partition lattice $\Pi_{n}$.

Let $T$ be a tree in $T_{n}$ with inner vertices $t_{1}, \ldots, t_{k}$. We denote the set of leaves in $T$ below an inner vertex $t$ by $\ell(t)$. We associate a nested set $\mathscr{Y}(T)$ in $\mathcal{N}\left(\Pi_{n}, \mathscr{I}\right)$ to $T$ by defining

$$
\mathscr{S}(T):=\left\{\ell\left(t_{i}\right) \mid i=1, \ldots, k\right\} .
$$

Conversely, let $\mathscr{S}=\left\{S_{1}, \ldots, S_{k}\right\}$ be a (reduced) nested set in $\Pi_{n}$ with respect to $\Im$. We define a rooted tree $\widetilde{T}(\mathscr{S})$ on the vertex set $\mathscr{S} \cup\{R\}$, where $R$ will be the root of the tree. Cover relations are defined by setting $S>T$ if and only if $T \in \max \mathscr{S}_{<S}$, and requiring the root $R$ to cover any element in $\max \mathscr{S}$. Observe that we allow the "root" of $\widetilde{T}(\mathscr{Y})$ to have degree 1 in this intermediate stage of the construction.

To obtain a tree with $n$ leaves, i.e., a simplex in $T_{n}$, we need to "grow leaves" on $\widetilde{T}(\mathscr{S})$. To do so, expand any leaf $S$ of $\widetilde{T}(\mathscr{S})$ into $|S|$ many leaves labeled with the elements in $S$. For every internal vertex $T$ of $\widetilde{T}(\mathscr{S})$ add leaves labeled with the elements of $T \backslash \bigcup_{S \in \mathscr{S}, S<T} S$, analogously, add leaves labeled with $[n] \backslash \bigcup \mathscr{S}$ to the root $R$. Denote the resulting tree by $T(\mathscr{S})$.

The two maps are inverse to each other, hence we have an (order-preserving) bijection between the faces of $T_{n}$ and the reduced minimal nested set complex 
$\mathcal{N}\left(\Pi_{n}, \mathscr{I}\right)$ of the partition lattice. We see that $T_{n}$ and $\mathcal{N}\left(\Pi_{n}, \mathscr{I}\right)$, in fact, are identical as abstract simplicial complexes.

Corollary 3.2. The order complex of the partition lattice $\Delta\left(\Pi_{n}\right)$ can be obtained from the complex of trees $T_{n}$ by a sequence of stellar subdivisions.

Proof. Referring to [Feichtner and Müller 2005, Theorem 4.2], we see that the order complex $\Delta\left(\Pi_{n}\right)$, i.e., the reduced maximal nested set complex $\mathcal{N}\left(\Pi_{n}, \Pi_{n}\right)$ of $\Pi_{n}$, can be obtained from the complex of trees $T_{n}=\mathcal{N}\left(\Pi_{n}, \mathscr{T}\right)$ by a sequence of stellar subdivisions.

Explicitly, the subdivision is given by the choice of a linear extension order on $\left(\Pi_{n} \backslash \mathscr{I}\right)^{\mathrm{op}}$, and by performing stellar subdivisions in simplices $F_{\mathscr{g}}(X)$ for poset elements $X$ in $\left(\Pi_{n} \backslash \mathscr{I}\right)^{\text {op }}$ along the given linear order.

Both the complex of trees and the partition lattice carry a natural action of the symmetric group $\Sigma_{n}$, which induces a $\Sigma_{n}$-action on the respective homology, each concentrated in top dimension (compare [Robinson and Whitehouse 1996] for the complex of trees, and [Stanley 1982] for the partition lattice). We recover the well-known isomorphism of $\Sigma_{n}$-modules in

Corollary 3.3. The top degree homology groups of $T_{n}$ and of $\Pi_{n}$ are isomorphic as $\Sigma_{n}$-modules:

$$
\widetilde{H}_{n-3}\left(T_{n}\right) \cong \Sigma_{n} \widetilde{H}_{n-3}\left(\Pi_{n}\right) .
$$

Proof. The maps defined in the proof of Theorem 3.1 are $\Sigma_{n}$-equivariant, hence they induce a $\Sigma_{n}$-module isomorphism in homology. Moreover, the subdivision referred to in the proof of Corollary 3.2 is $\Sigma_{n}$-equivariant, where the action on $\Delta\left(\Pi_{n}\right)$ comes from the action of $\Sigma_{n}$ on $\Pi_{n}$. Observe that not any individual subdivision step is equivariant, but simultaneous subdivisions for a fixed partition type are.

Remarks 3.4. (1) In recent work, Ardila and Klivans showed that the complex of trees $T_{n}$ coincides as a simplicial complex with the matroid stratification of the Bergman complex of the graphical matroid $M\left(K_{n}\right)$ [Ardila and Klivans 2006, Section 3]. This implies that $\Delta\left(\mathscr{L}\left(M\left(K_{n}\right)\right)\right)=\Delta\left(\Pi_{n}\right)$ subdivides the complex of trees, in fact, the order complex coincides with the weight stratification of the Bergman complex. Our result gives an explicit sequence of stellar subdivisions relating one complex to the other. Independently, Ardila (personal communication, 2004) has described a sequence of subdivisions connecting $T_{n}$ and $\Delta\left(\Pi_{n}\right)$ which remains to be compared to the one presented here.

(2) For an arbitrary matroid $M$, the matroid stratification of the Bergman complex $B(M)$ can be subdivided so as to realize the order complex of the lattice of flats $\mathscr{L}(M)$ [Ardila and Klivans 2006, Theorem 1]. This stratification, in fact, is 


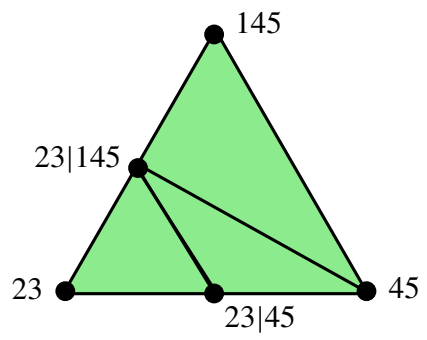

Figure 2. Subdivision of $\mathscr{T}=\{23,45,145\}$ in $\Delta\left(\Pi_{5}\right)$.

the finest in a family of subdivisions having the combinatorics of reduced nested set complexes $\mathcal{N}(\mathscr{L}(M), \mathscr{G})$, the coarsest of which, corresponding to $\mathcal{N}(\mathscr{L}(M), \mathscr{I})$, still subdivides the matroid stratification of $B(M)$ [Feichtner and Sturmfels 2005].

Our theorem implies that for the particular case of the graphic matroid $M\left(K_{n}\right)$ the coarsest nested set stratification coincides with the matroid stratification of the Bergman complex. This is not true in general; for an example as well as a characterization of when the stratifications coincide, see [Feichtner and Sturmfels 2005, Example 1.2 and Theorem 5.3].

(3) The subdivision of $T_{n}$ described in Corollary 3.2 can not be connected to the barycentric subdivision of the complex of trees, $\operatorname{bsd}\left(T_{n}\right)$, by further stellar subdivisions. In earlier work, M. Wachs (personal communication, 2004) related cells in $T_{n}$ to simplices in the barycentric subdivision of $T_{n}$ in a way that suggested such a connection.

As an example, let us observe that the subdivision $\Delta\left(\Pi_{5}\right)$ of $T_{5}$ is not refined by $\operatorname{bsd}\left(T_{5}\right)$ :

Consider the triangle $\mathscr{T}=\{23,45,145\}$ in $\mathcal{N}\left(\Pi_{5}, \mathscr{T}\right)=T_{5}$. The sequence of stellar subdivisions found in Corollary 3.2 has to be nonincreasing on nonbuilding set elements, hence, in the course of subdividing $T_{5}$, we first do a stellar subdivision in the edge $(23,145)$, and later in the edge $(23,45)$. The resulting subdivision of $\mathscr{T}$ is depicted in Figure 2. The edge $(23|45<23| 145)$ in $\Delta\left(T_{5}\right)$ is not refined by the barycentric subdivision of $\mathscr{T}$.

\section{A cohomology basis for the complex of trees}

We start this section by recalling some standard constructions associated with geometric lattices. For standard matroid terminology used throughout, we refer to [Oxley 1992].

Let $\mathscr{L}$ be a geometric lattice of rank $r$ with a fixed linear order $\omega$ on its set of atoms $\mathfrak{A}(\mathscr{L})$. A broken circuit in $\mathscr{L}$ is a subset $D$ of $\mathfrak{A}(\mathscr{L})$ that can be written as a circuit in $\mathscr{L}$ with its minimal element removed, $D=C \backslash \min C, C$ a circuit in $\mathscr{L}$. 
The maximal independent sets in $\mathscr{L}$ that do not contain any broken circuits are known as the no broken circuit bases of $\mathscr{L}$, denoted by $\mathbf{n b c}_{\omega}(\mathscr{L})$. The no broken circuit bases play an important role for understanding the (co)homology of $\Delta(\mathscr{L})$.

There is a standard labeling $\lambda$ of cover relations $X>Y$ in $\mathscr{L}$. For $X \in \mathscr{L}$, let $\lfloor X\rfloor:=\{A \in \mathfrak{A}(\mathscr{L}) \mid A \leq X\}$, the set of atoms in $\mathscr{L}$ below $X$, and define

$$
\lambda(X>Y):=\min (\lfloor X\rfloor \backslash\lfloor Y\rfloor) .
$$

This labeling in fact is an $E L$-labeling of $\mathscr{L}$ in the sense of [Björner and Wachs 1983], thus, by ordering maximal chains lexicographically, it induces a shelling of the order complex $\Delta(\mathscr{L})$. Denote by $\mathrm{dc}_{\omega}(\mathscr{L})$ the set of maximal chains in $\mathscr{L}$ with (strictly) decreasing label sequence:

$\operatorname{dc}_{\omega}(\mathscr{L}):=\left\{\hat{0}<c_{1}<\cdots<c_{r-1}<\hat{1} \mid \lambda\left(c_{1}>\hat{0}\right)>\lambda\left(c_{2}>c_{1}\right)>\cdots>\lambda\left(\hat{1}>c_{r-1}\right)\right\}$.

The characteristic cohomology classes $\left[c^{*}\right]$ for $c \in \mathbf{d c}_{\omega}(\mathscr{L})$, i.e., classes represented by cochains that evaluate to 1 on $c$ and to 0 on any other top-dimensional simplex of $\Delta(\mathscr{L})$, form a basis of the only nonzero reduced cohomology group of the order complex, $\widetilde{H}^{r-1}(\Delta(\mathscr{L}))$.

We add the notion of proper maximal nested sets to the standard notions for geometric lattices with fixed atom order that we listed so far. The concept has appeared in [De Concini and Procesi 2005]. Define a map $\phi: \mathscr{I} \rightarrow \mathfrak{A}(\mathscr{L})$ by setting $\phi(S):=\min \lfloor S\rfloor$ for $S \in \mathscr{I}$. A maximal nested set $\mathscr{S}$ in the (nonreduced) nested set complex $\widetilde{\mathcal{N}}(\mathscr{L}, \mathscr{I})$ is called proper if the set $\{\phi(S) \mid S \in \mathscr{S}\}$ is a basis of $\mathscr{L}$. Denote the set of proper maximal nested sets in $\mathscr{L}$ by $\mathbf{p n}_{\omega}(\mathscr{L})$.

We define maps connecting $\mathbf{n b c}_{\omega}(\mathscr{L})$, $\mathbf{d c}_{\omega}(\mathscr{L})$, and $\mathbf{p n}_{\omega}(\mathscr{L})$ for a given geometric lattice $\mathscr{L}$. In the following proposition we will see that these maps provide bijective correspondences between the respective sets.

To begin with, define $\Psi: \mathbf{n b c}_{\omega}(\mathscr{L}) \rightarrow \mathbf{d c}_{\omega}(\mathscr{L})$ by

$$
\Psi\left(a_{1}, \ldots, a_{r}\right)=\left(\hat{0}<a_{r}<a_{r} \vee a_{r-1}<\cdots<a_{r} \vee a_{r-1} \vee \cdots \vee a_{1}=\hat{1}\right),
$$

where the $a_{1}, \ldots, a_{r}$ are assumed to be in ascending order with respect to $\omega$.

Next, define $\Theta: \mathbf{d c}_{\omega}(\mathscr{L}) \rightarrow \mathbf{p n}_{\omega}(\mathscr{L})$ by

$$
\Theta\left(\hat{0}<c_{1}<\cdots<c_{r-1}<\hat{1}\right)=F\left(c_{1}\right) \cup F\left(c_{2}\right) \cup \cdots \cup F\left(c_{r-1}\right) \cup F(\hat{1}),
$$

for a chain $c: \hat{0}<c_{1}<\cdots<c_{r-1}<\hat{1}$ in $\mathscr{L}$ with decreasing label sequence, where $F\left(c_{i}\right)$ denotes the set of factors of $c_{i}$ with respect to the minimal building set $\mathscr{I}$ in $\mathscr{L}$.

Finally, define $\Phi: \mathbf{p n}_{\omega}(\mathscr{L}) \rightarrow \mathbf{n b c}_{\omega}(\mathscr{L})$ by

$$
\Phi(\mathscr{Y})=\{\phi(S) \mid S \in \mathscr{Y}\},
$$

for $\mathscr{Y} \in \mathbf{p n}_{\omega}(\mathscr{L})$. 
Example 4.1. Consider the partition lattice $\Pi_{5}$ with lexicographic order lex on its set of atoms $i j, 1 \leq i<j \leq 5$. For $b=\{12,14,23,45\} \in \mathbf{n b c}_{\text {lex }}\left(\Pi_{5}\right)$ we have

$$
\Psi(b)=(0<45<23|45<23| 145<\hat{1}) .
$$

Observe that the chain is constructed by taking consecutive joins of elements in the opposite of the lexicographic order.

Going further using $\Theta$ we obtain the following proper nested set,

$$
\begin{aligned}
\Theta(0<45<23|45<23| 145<\hat{1}) & =\{45\} \cup\{23,45\} \cup\{23,145\} \cup\{12345\} \\
& =\{23,45,145,12345\} .
\end{aligned}
$$

Applying $\Phi$ we retrieve the no broken circuit basis we started with:

$$
\Phi(\{23,45,145,12345\})=\{12,23,14,45\} .
$$

Proposition 4.2. For a geometric lattice $\mathscr{L}$ with a given linear order $\omega$ on its atoms the maps $\Psi, \Theta$, and $\Phi$ defined above give bijective correspondences between (1) the no broken circuit bases $\mathbf{n b c}_{\omega}(\mathscr{L})$ of $\mathscr{L},(2)$ the maximal chains in $\mathscr{L}$ with decreasing label sequence, $\mathbf{d c}_{\omega}(\mathscr{L})$, and (3) the proper maximal nested sets $\mathbf{p n}_{\omega}(\mathscr{L})$ in $\widetilde{\mathcal{N}}(\mathscr{L}, \mathscr{I})$, respectively.

Proof. The map $\Psi: \mathbf{n b c} \mathbf{c}_{\omega}(\mathscr{L}) \rightarrow \mathbf{d c}_{\omega}(\mathscr{L})$ is well known in the theory of geometric lattices. It is the standard bijection relating no broken circuit bases to cohomology generators of the lattice; compare [Björner 1992, Section 7.6] for details. The composition of maps $\eta:=\Theta \circ \Psi: \mathbf{n b c}_{\omega}(\mathscr{L}) \rightarrow \mathbf{p n}_{\omega}(\mathscr{L})$ is shown to be a bijection with inverse $\Phi: \mathbf{p n}_{\omega}(\mathscr{L}) \rightarrow \mathbf{n b c}_{\omega}(\mathscr{L})$; see [De Concini and Procesi 2005, Theorem 2.2]. This implies that $\Theta: \mathbf{d c}_{\omega}(\mathscr{L}) \rightarrow \mathbf{p n}_{\omega}(\mathscr{L})$ is bijective as well, which completes the proof of our claim.

The aim of the next proposition is to trace the support simplices for the cohomology bases $\left\{\left[c^{*}\right] \mid c \in \mathbf{d c}_{\omega}\left(\Pi_{n}\right)\right\}$ of $\Delta\left(\Pi_{n}\right)$ through the inverse stellar subdivisions linking $\Delta\left(\Pi_{n}\right)$ to the complex of trees $T_{n}=\mathcal{N}\left(\Pi_{n}, \mathscr{P}\right)$. For the moment we can stay with the full generality of geometric lattices and study support simplices for maximal simplices of $\Delta(\mathscr{L})$ in the minimal reduced nested set complex $\mathcal{N}(\mathscr{L}, \mathscr{I})$.

Proposition 4.3. Let $\mathscr{L}$ be a geometric lattice, $c: c_{1}<\ldots<c_{r-1}$ a maximal simplex in $\Delta(\mathscr{L})$. The maximal simplex in $\mathcal{N}(\mathscr{L}, \mathscr{I})$ supporting $c$ is given by the union of sets of factors

$$
F\left(c_{1}\right) \cup F\left(c_{2}\right) \cup \cdots \cup F\left(c_{r-1}\right) .
$$

Proof. There is a sequence of building sets

$$
\mathscr{L}=\mathscr{G}_{1} \supseteq \mathscr{G}_{2} \supseteq \cdots \supseteq \mathscr{G}_{t}=\mathscr{I},
$$


connecting $\mathscr{L}$ and $\mathscr{I}$ which is obtained by removing elements of $\mathscr{L} \backslash \mathscr{I}$ from $\mathscr{L}$ in nondecreasing order: $\mathscr{G}_{i} \backslash \mathscr{G}_{i+1}=\left\{G_{i}\right\}$ with $G_{i}$ minimal in $\mathscr{G}_{i} \backslash \mathscr{I}$ for $i=$ $1, \ldots, t-1$. The corresponding nested set complexes are linked by inverse stellar subdivisions:

$$
\mathcal{N}\left(\varphi_{i}\right)=\operatorname{st}\left(\mathcal{N}\left(\varphi_{i+1}\right), V\left(F_{\mathscr{\Phi}}\left(G_{i}\right)\right)\right), \quad \text { for } i=1, \ldots t-1,
$$

where $V\left(F_{\mathcal{I}}\left(G_{i}\right)\right)$ denotes the simplex in $\mathcal{N}\left(G_{i+1}\right)$ spanned by the factors of $G_{i}$ with respect to the minimal building set $\mathscr{S}$.

We trace what happens to the support simplex of $c$ along the sequence of inverse stellar subdivisions connecting $\Delta(\mathscr{L})$ with $\mathcal{N}(\mathscr{F})$. The support simplex of $c$ remains unchanged in step $i$ unless $G_{i}$ coincides with a (reducible) chain element $c_{j}$ (the irreducible chain elements can be replaced any time by their "factors": $F\left(c_{k}\right)=\left\{c_{k}\right\}$ for $\left.c_{k} \in \mathscr{I}\right)$.

We can assume that the support simplex of $c$ in $\mathcal{N}\left(\mathscr{G}_{i}\right)$ is of the form

$$
\mathscr{S}=F\left(c_{1}\right) \cup \cdots \cup F\left(c_{j-1}\right) \cup\left\{c_{j}\right\} \cup \cdots \cup\left\{c_{r-1}\right\},
$$

and we aim to show that the support simplex of $c$ in $\mathcal{N}\left(\varphi_{i+1}\right)$ is given by

$$
\mathscr{T}=F\left(c_{1}\right) \cup \cdots \cup F\left(c_{j-1}\right) \cup F\left(c_{j}\right) \cup\left\{c_{j+1}\right\} \cup \cdots \cup\left\{c_{r-1}\right\} .
$$

Recall that the respective face posets of the nested set complexes are connected by a combinatorial blowup

$$
\mathscr{F}\left(\mathcal{N}\left(\varphi_{i}\right)\right)=\mathrm{Bl}_{F\left(G_{i}\right)}\left(\mathscr{F}\left(\mathcal{N}\left(\varphi_{i+1}\right)\right)\right) .
$$

See [Feichtner and Kozlov 2004, 3.1] for the concept of a combinatorial blowup in meet semilattices. Hence, the support simplex $\mathscr{Y}$ of $c$ in $\mathcal{N}\left(\mathscr{G}_{i}\right)$ is of the form $\mathscr{S}=\mathscr{Y}_{0} \cup\left\{c_{j}\right\}$ with $\mathscr{Y}_{0} \in \mathcal{N}\left(\mathscr{G}_{i+1}\right)$ (it is an element in the "copy" of the lower ideal of elements in $\mathscr{F}\left(\mathcal{N}\left(\varphi_{i+1}\right)\right)$ having joins with $\left.F\left(G_{i}\right)\right)$. Due to (3) we know that $\mathscr{Y}_{0} \nsupseteq F\left(G_{i}\right)$ and $\mathscr{S}_{0} \cup F\left(G_{i}\right) \in \mathcal{N}\left(\mathscr{G}_{i+1}\right)$, which in fact is the new support simplex of $c$. Let us mention in passing that, since we are talking about maximal simplices, $\mathscr{Y}_{0}$ contains $F\left(G_{i}\right)$ up to exactly one element $X_{i} \in \mathscr{L}$.

Since $c_{j}$ is not contained in any of the $F\left(c_{i}\right), i=1, \ldots, j-1$, we have

$$
\mathscr{S}_{0}=F\left(c_{1}\right) \cup \cdots \cup F\left(c_{j-1}\right) \cup\left\{c_{j+1}\right\} \cup \cdots \cup\left\{c_{r-1}\right\},
$$

and we find that $\mathcal{T}=F\left(c_{1}\right) \cup \cdots \cup F\left(c_{j-1}\right) \cup F\left(c_{j}\right) \cup\left\{c_{j+1}\right\} \cup \cdots \cup\left\{c_{r-1}\right\}$ as claimed.

Example 4.4. Let us again consider the partition lattice $\Pi_{5}$. The support simplex of $c: 45<23|45<23| 145$ in $\mathcal{N}\left(\Pi_{5}, \mathscr{I}\right)$ is $\{23,45,145\}$. We depict in Figure 3 how the support simplex of $c$ changes in the sequence of inverse stellar subdivisions from $\Delta\left(\Pi_{5}\right)$ to $\mathcal{N}\left(\Pi_{5}, \mathscr{I}\right)$. 


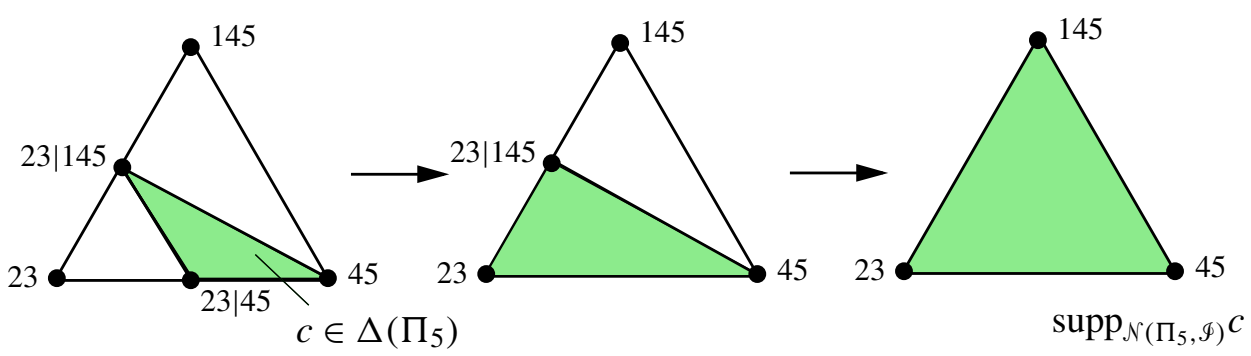

Figure 3. Support simplices of $c$.

We now combine our findings to provide an explicit cohomology basis for the complex of trees $T_{n}$.

We call a binary rooted tree $T$ with $n$ leaves labeled $1, \ldots, n$ admissible, if, when recording the 2 nd smallest label on the sets of leaves below any of the $n-1$ nonleaves of $T$, we find each of the labels $2, \ldots, n$ exactly once. For an example of an admissible tree in $T_{5}$ see Figure 4.

Proposition 4.5. The characteristic cohomology classes associated with admissible trees in $T_{n}$,

$$
\left\{\left[T^{*}\right] \in \widetilde{H}^{n-3}\left(T_{n}\right) \mid T \text { admissible in } T_{n}\right\},
$$

form a basis for the (reduced) cohomology of the complex of trees $T_{n}$.

Proof. We set out from the linear basis for $\widetilde{H}^{n-3}\left(\Delta\left(\Pi_{n}\right)\right)$ provided by characteristic cohomology classes associated with the decreasing chains $\mathbf{d c}_{\omega}\left(\Pi_{n}\right)$ in $\Pi_{n}$. Combining Proposition 4.3 with the definition of the bijection $\Theta: \mathbf{d c}_{\omega}\left(\Pi_{n}\right) \rightarrow \mathbf{p n}_{\omega}\left(\Pi_{n}\right)$ in (1) we find that the characteristic cohomology classes associated with (reduced) proper maximal nested sets $\mathbf{p n}_{\omega}\left(\Pi_{n}\right)$ provide a linear basis for $\widetilde{H}^{n-3}\left(\mathcal{N}\left(\Pi_{n}, \mathscr{I}\right)\right)$. We tacitly make use of the bijection between maximal simplices in $\widetilde{\mathcal{N}}(\mathscr{L}, \mathscr{I})$ and $\mathcal{N}(\mathscr{L}, \mathscr{I})$ given by removing the maximal element $\hat{1}$ of $\mathscr{L}$.

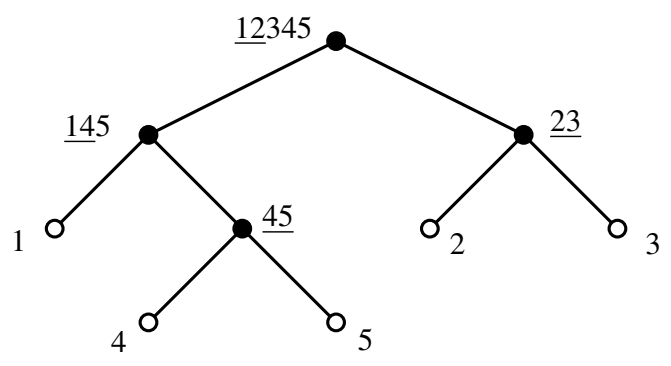

Figure 4. An admissible tree in $T_{5}$. 
To describe support simplices explicitly, recall that proper maximal nested sets are inverse images of no broken circuit bases under $\Phi: \mathbf{p n}_{\omega}\left(\Pi_{n}\right) \rightarrow \mathbf{n b c}_{\omega}\left(\Pi_{n}\right)$ as defined in (2). The no broken circuit bases of $\Pi_{n}$ with respect to the lexicographic order on atoms, i.e., on pairs $(i, j), 1 \leq i<j \leq n$, are $(n-1)$-element subsets of the form

$$
(1,2),\left(i_{2}, 3\right), \ldots,\left(i_{n-1}, n\right)
$$

with $1 \leq i_{j} \leq j$ for $j=2, \ldots, n-1$. Inverse images under $\Phi$ are maximal nested sets $\mathscr{S} \in \widetilde{\mathcal{N}}\left(\Pi_{n}, \mathscr{I}\right)$ such that $\{\phi(S) \mid S \in \mathscr{Y}\}$ gives collections of pairs with each integer from 2 to $n$ occurring exactly once in the second coordinate. Applying the isomorphism between $\mathcal{N}\left(\Pi_{n}, \mathscr{Y}\right)$ and $T_{n}$ from Theorem 3.1 shows that the characteristic cohomology classes on admissible trees in $T_{n}$ indeed form a basis of $\widetilde{H}^{n-3}\left(T_{n}\right)$.

Remark 4.6. Our basis of admissible trees differs from the one presented in [Trappmann and Ziegler 1998, Corollary 5] as a consequence of their shelling argument for complexes of trees.

\section{Complexes of $k$-trees and other generalizations}

The intriguing representation theory of complexes of trees $T_{n}$ in [Robinson and Whitehouse 1996] has given rise in [Hanlon 1996] to a generalization to complexes of $k$-trees.

Definition 5.1. The complex of $k$-trees $T_{n}^{(k)}, n \geq 1, k \geq 1$, is the abstract simplicial complex with faces corresponding to combinatorial types of rooted trees with $(n-1) k+1$ leaves labeled $1, \ldots,(n-1) k+1$, with all outdegrees at least $k+1$ and congruent to 1 modulo $k$, and at least one internal edge. The partial order among the rooted trees is given by contraction of internal edges.

Alternatively, we could define $T_{n}^{(k)}$ as the simplicial complex with faces corresponding to (nonrooted) trees with $(n-1) k+2$ labeled leaves, all degrees of nonleaves at least $k+2$ and congruent to 2 modulo $k$, and at least one internal edge. Again, the order relation is given by contracting internal edges. Observe that for $k=1$ we recover the complex of trees $T_{n}$. The face poset of our complex $T_{n}^{(k)}$ is the poset $\mathscr{L}_{n-1}^{(k)}$ of [Hanlon 1996].

The complexes $T_{n}^{(k)}$ are pure simplicial complexes of dimension $n-3$. They are Cohen-Macaulay [Hanlon 1996, Theorem 2.3]; later, as mentioned in the introduction for the special case $k=1$, a shellability result was obtained by Trappmann and Ziegler and, independently, by Wachs.

The complexes $T_{n}^{(k)}$ carry a natural $\Sigma_{N}$-action for $N=(n-1) k+1$ by permutation of leaves, which induces a $\Sigma_{N}$-action on top degree homology. It follows from [Hanlon 1996, Theorem 1.1; Hanlon and Wachs 1995, Theorems 3.11 and 
4.13] that $\widetilde{H}_{n-3}\left(T_{n}^{(k)}\right)$ is isomorphic as an $\Sigma_{N}$-module to $\widetilde{H}_{n-3}\left(\Pi_{N}^{(k)}\right)$, where $\Pi_{N}^{(k)}$ is the subposet of $\Pi_{N}$ consisting of all partitions with block sizes congruent 1 modulo $k$. The poset $\Pi_{N}^{(k)}$ had been studied before on its own right: it was shown to be Cohen-Macaulay in [Björner 1980], its homology and $\Sigma_{N}$-representation theory was studied by Calderbank, Hanlon and Robinson [Calderbank et al. 1986]. In fact, both $\Sigma_{N}$-modules $\widetilde{H}_{n-3}\left(T_{n}^{(k)}\right)$ and $\widetilde{H}_{n-3}\left(\Pi_{N}^{(k)}\right)$ are isomorphic to the $1^{N}$ homogeneous piece of the free Lie $k$-algebra constructed in [Hanlon and Wachs 1995].

This certainly provides enough evidence to look for a topological explanation of the isomorphism of $\Sigma_{N}$-modules:

Question 5.2. Is the complex of $k$-trees $T_{n}^{(k)}$ related to the order complex of $\Pi_{(n-1) k+1}^{(k)}$ in the same way as $T_{n}$ is related to the order complex of $\Pi_{n}$, i.e., is $T_{n}^{(k)}$ homeomorphic to $\Delta\left(\Pi_{(n-1) k+1}^{(k)}\right)$ ? More than that, can $\Delta\left(\Pi_{(n-1) k+1}^{(k)}\right)$ be obtained from $T_{n}^{(k)}$ by a sequence of stellar subdivisions?

An approach to this question along the lines of Section 3 does not work right away: The poset $\Pi_{(n-1) k+1}^{(k)}$ is not a lattice, and a concept of nested sets for more general posets is not (yet) at hand.

The generalization of $T_{n}$ to complexes of $k$-trees $T_{n}^{(k)}$ thus turns out to be somewhat unnatural from the point of view of nested set constructions. We propose another generalization which is motivated by starting with a natural generalization of the partition lattice that remains within the class of lattices.

Definition 5.3. For $n>k \geq 2$, the $k$-equal lattice $\Pi_{n, k}$ is the sublattice of the partition lattice $\Pi_{n}$ that is join-generated by partitions with a single nontrivial block of size $k$.

Observe that we retrieve $\Pi_{n}$ for $k=2$. There is an extensive study of the $k$-equal lattice in the literature, mostly motivated by the fact that $\Pi_{n, k}$ is the intersection lattice of a natural subspace arrangement, the k-equal arrangement. Its homology has been calculated in [Björner and Welker 1995], it was shown to be shellable in [Björner and Wachs 1996], and its $\Sigma_{n}$-representation theory has been studied in [Sundaram and Wachs 1997].

The irreducibles $\mathscr{I}$ in $\Pi_{n, k}$ are partitions with exactly one nontrivial block, this time of size at least $k$. Sets of irreducibles are nested if and only if for any two elements the nontrivial blocks are either contained in one another or disjoint.

Constructing trees from nested sets, analogous to the construction of $T_{n}$ from $\mathcal{N}\left(\Pi_{n}, \mathscr{T}\right)$ in the proof of Theorem 3.1, suggests the following definition:

Definition 5.4. The complex of k-equal trees $T_{n, k}$ is a simplicial complex with maximal simplices given by combinatorial types of rooted trees $T$ on $n$ labeled 
leaves which are binary except at preleaves, where they are $k$-ary. Here, preleaves of $T$ are leaves of the tree that is obtained from $T$ by removing the leaves. Lowerdimensional simplices are obtained by contracting internal edges.

We depict the tree types occurring as maximal simplices of $T_{7,3}$ in Figure 5. Observe that one is a 3-dimensional simplex in $T_{7,3}$, whereas the two others are 2-dimensional. The definition of $T_{n, k}$ does not appeal as natural, however, it is a trade off for the following Proposition and Corollary which are obtained literally for free, having the arguments of Section 3 at hand.
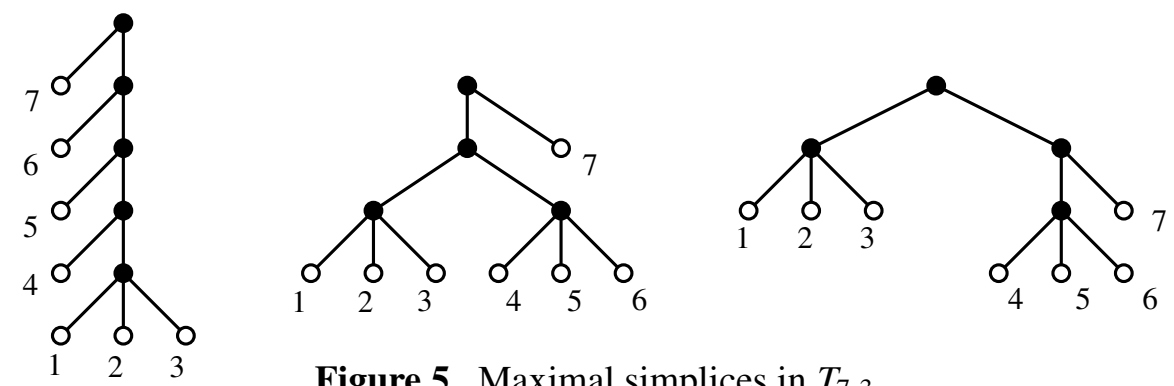

Figure 5. Maximal simplices in $T_{7,3}$

Proposition 5.5. The complex of $k$-equal trees, $T_{n, k}$, and the minimal nested set complex of the $k$-equal lattice, $\mathcal{N}\left(\Pi_{n, k}, \mathscr{I}\right)$, coincide as abstract simplicial complexes. In particular, the order complex $\Delta\left(\Pi_{n, k}\right)$ can be obtained from $T_{n, k}$ by a sequence of stellar subdivisions.

Proof. There is a bijection between trees in $T_{n, k}$ and nested sets in $\mathcal{N}\left(\Pi_{n, k}, \mathscr{Y}\right)$ analogous to the bijection between $T_{n}$ and $\mathcal{N}\left(\Pi_{n}, \mathscr{I}\right)$ that we described in the proof of Theorem 3.1. Referring again to [Feichtner and Müller 2005, Theorem 4.2], the complexes are connected by a sequence of stellar subdivisions.

Corollary 5.6. The graded homology groups of the complex of k-equal trees and of the $k$-equal lattice are isomorphic as $\Sigma_{n}$-modules:

$$
\widetilde{H}_{*}\left(T_{n, k}\right) \cong \Sigma_{n} \widetilde{H}_{*}\left(\Pi_{n, k}\right)
$$

\section{Note added in proof}

Question 5.2 has been answered affirmatively by Delucchi [2005].

\section{Acknowledgments}

I thank Michelle Wachs and Federico Ardila for stimulating discussions at the IAS/Park City Mathematics Institute in July 2004.

This work was carried out while the author was at the Swiss Federal Institute of Technology (ETH), Zurich, and at the Mathematical Sciences Research Institute, Berkeley. 


\section{References}

[Ardila and Klivans 2006] F. Ardila and C. J. Klivans, "The Bergman complex of a matroid and phylogenetic trees”, J. Combin. Theory Ser. B 96:1 (2006), 38-49. MR 2006i:05034 Zbl 1082.05021

[Billera et al. 2001] L. J. Billera, S. P. Holmes, and K. Vogtmann, "Geometry of the space of phylogenetic trees", Adv. in Appl. Math. 27:4 (2001), 733-767. MR 2002k:05229 Zbl 0995.92035

[Björner 1980] A. Björner, "Shellable and Cohen-Macaulay partially ordered sets", Trans. Amer. Math. Soc. 260:1 (1980), 159-183. MR 81i:06001 Zbl 0441.06002

[Björner 1992] A. Björner, "The homology and shellability of matroids and geometric lattices", pp. 226-283 in Matroid applications, edited by N. White, Encyclopedia Math. Appl. 40, Cambridge Univ. Press, Cambridge, 1992. MR 94a:52030 Zbl 0772.05027

[Björner and Wachs 1983] A. Björner and M. Wachs, "On lexicographically shellable posets", Trans. Amer. Math. Soc. 277:1 (1983), 323-341. MR 84f:06004 Zbl 0514.05009

[Björner and Wachs 1996] A. Björner and M. Wachs, "Shellable nonpure complexes and posets, I", Trans. Amer. Math. Soc. 348:4 (1996), 1299-1327. MR 96i:06008 Zbl 0857.05102

[Björner and Welker 1995] A. Björner and V. Welker, "The homology of " $k$-equal" manifolds and related partition lattices", Adv. Math. 110:2 (1995), 277-313. MR 95m:52029 Zbl 0845.57020

[Boardman 1971] J. M. Boardman, "Homotopy structures and the language of trees", pp. 37-58 in Algebraic topology (Madison, WI, 1970), edited by A. Liulevicius, Proc. Sympos. Pure Math. 22, Amer. Math. Soc., Providence, RI, 1971. MR 50 \#3215 Zbl 0242.55012

[Calderbank et al. 1986] A. R. Calderbank, P. Hanlon, and R. W. Robinson, "Partitions into even and odd block size and some unusual characters of the symmetric groups", Proc. London Math. Soc. (3) 53:2 (1986), 288-320. MR 87m:20042 Zbl 0602.20017

[De Concini and Procesi 1995] C. De Concini and C. Procesi, "Wonderful models of subspace arrangements”, Selecta Math. (N.S.) 1:3 (1995), 459-494. MR 97k:14013 Zbl 0842.14038

[De Concini and Procesi 2005] C. De Concini and C. Procesi, "Nested sets and Jeffrey-Kirwan residues", pp. 139-149 in Geometric methods in algebra and number theory, edited by F. Bogomolov and Y. Tschinkel, Progress in Math. 235, Birkhäuser, Boston, 2005. MR 2006j:32032 Zbl 1093.52503

[Delucchi 2005] E. Delucchi, "Subdivision of complexes of $k$-trees", preprint, ETH Zurich, 2005. math.CO/0509378

[Feichtner and Kozlov 2004] E.-M. Feichtner and D. N. Kozlov, "Incidence combinatorics of resolutions”, Selecta Math. (N.S.) 10:1 (2004), 37-60. MR MR2061222 Zb1 1068.06004

[Feichtner and Müller 2005] E. M. Feichtner and I. Müller, "On the topology of nested set complexes”, Proc. Amer. Math. Soc. 133:4 (2005), 999-1006. MR 2006c:06005 Zbl 1053.05125

[Feichtner and Sturmfels 2005] E. M. Feichtner and B. Sturmfels, "Matroid polytopes, nested sets and Bergman fans”, Port. Math. (N.S.) 62:4 (2005), 437-468. MR 2006j:05036 Zbl 1092.52006

[Feichtner and Yuzvinsky 2004] E. M. Feichtner and S. Yuzvinsky, "Chow rings of toric varieties defined by atomic lattices", Invent. Math. 155:3 (2004), 515-536. MR 2004k:14009 Zbl 1083.14059

[Hanlon 1996] P. Hanlon, "Otter's method and the homology of homeomorphically irreducible $k$ trees", J. Combin. Theory Ser. A 74:2 (1996), 301-320. MR 98g:05155 Zbl 0848.05021

[Hanlon and Wachs 1995] P. Hanlon and M. Wachs, "On Lie k-algebras", Adv. Math. 113:2 (1995), 206-236. MR 96h:17006 Zbl 0844.17001

[Oxley 1992] J. G. Oxley, Matroid theory, Oxford University Press, New York, 1992. MR 94d:05033 Zbl 0784.05002 
[Robinson and Whitehouse 1996] A. Robinson and S. Whitehouse, "The tree representation of $\Sigma_{n+1} ”$, J. Pure Appl. Algebra 111:1-3 (1996), 245-253. MR 97g:55010 Zbl 0865.55010

[Stanley 1982] R. P. Stanley, "Some aspects of groups acting on finite posets", J. Combin. Theory Ser. A 32:2 (1982), 132-161. MR 83d:06002 Zbl 0496.06001

[Sundaram and Wachs 1997] S. Sundaram and M. Wachs, "The homology representations of the $k$ equal partition lattice”, Trans. Amer. Math. Soc. 349:3 (1997), 935-954. MR 97j:05063 Zbl 0863. 05082

[Trappmann and Ziegler 1998] H. Trappmann and G. M. Ziegler, "Shellability of complexes of trees”, J. Combin. Theory Ser. A 82:2 (1998), 168-178. MR 99f:05122 Zbl 0916.06004

[Vogtmann 1990] K. Vogtmann, "Local structure of some Out $\left(F_{n}\right)$-complexes", Proc. Edinburgh Math. Soc. (2) 33:3 (1990), 367-379. MR 92d:57002 Zbl 0694.20021

[Wachs 2003] M. Wachs, "Posets of graphs, partitions and trees", talk at Banff International Research Station, May 2003, Available at http://www.math.miami.edu/ wachs/talks.html. Based on joint work with John Shareshian.

Received February 5, 2005.

EVA MARIA FEICHTNER

DEPARTMENT OF MATHEMATICS

UNIVERSITY OF STUTTGART

70569 STUTTGART

GERMANY

feichtne@igt.uni-stuttgart.de 